Betriebsinterne Partizipation und Ökologie

\title{
Umweltschutz von innen
}

\section{Betrieblicher Umweltschutz wird effektiv durch Beteiligung und Kooperation der Beschäftigten. Grundvoraussetzungen dafür sind umfassende interne Kom- munikation, die Erhebung und Nutzung der entsprechenden Daten sowie die Ein- richfung von Beteiligungsmöglichkeiten. Trotz guter Absichten vieler Firmen ha- pert es kräftig an der Umsetzung.}

$\mathrm{E}$ Von Herbert Klemisch ffektiver betrieblicher Umweltschutz ist nur durch das Mitwirken aller Beschäftigten möglich. Deshalb wollen wir das Umweltbewusstsein unserer Mitarbeiter durch Informationen, Schulungen und Unterweisungen fördern. "Ähnlich positiv wie in den Umweltleitlinien der Firma Hipp ist der vermutete Grundtenor zum Zusammenhang von Ökologie und Partizipation, wenn man die Umwelt- und Nachhaltigkeitsberichte von Unternehmen unter die Lupe nimmt. Konfrontiert man betriebliche Akteure wie Geschäftsleitung, Betriebsrat oder Umweltbeauftragte mit dieser These, erhält man breite Zustimmung (1). Die Realität sieht in Klein- und Mittelständischen Unternehmen (KMU) allerdings oft anders aus.

\section{- Akteure des Betrieblichen Umweltschutzes}

Grundvoraussetzung für die Umsetzung eines Umweltmanagements ist die Zustimmung oder zumindest passive Unterstïtzung der Geschäftsleitung. Die wesentlichen Hemmnisse finden sich auf der mittleren Leitungsebene, bei Fachabteilungsleitern, Produktionsleitern, Meistern und Vorarbeitern sowie auf der Ebene der einfachen Mitarbeiter. Auf der mittleren Ebene wird der Einführung von UMS oder von Verbesserungsmaßnahmen im Umweltschutz vor allem aus Angst vor Macht- und Kompetenzverlusten Widerstand entgegengesetzt. Eine abteilungsübergreifende Vernetzung, wie sie die ökologische Modernisierung des Betriebs nahe legt, wird als Erosion gefestigter Besitzstände erlebt. Neue Beteiligungsformen wie Umweltausschüsse oder Öko-Teams werden als Aushöhlung von Verantwortungsbereichen begriffen. Die Enthierarchisierung im Zuge neuer Managementkonzepte kann darüber hinaus zur Wegrationalisierung von Arbeitsplätzen führen, was das Misstrauen gegenüber Neuerungen verstärkt. Diese Skepsis ist auch bei Betriebsräten festzustellen.
Bei den „,normalen“ Beschäftigten sind es Ängste vor zusätzlicher Arbeitsbelastung, der Infragestellung von Alltagsroutinen und der Unsicherheit gegenüber unbekannten Anforderungen, die die Innovationsfähigkeit der Gesamtorganisation behindern. Die gleichzeitige ökonomische, arbeitspolitische und ökologische Reorganisation eines Unternehmens kann strukturelle Unsicherheiten hervorrufen und Abwehrhaltungen provozieren. Zentraler Akteur ist für alle Betriebe mit einem UMS der/die Umweltmanagementbeauftragte (UMB). An sie werden hohe Erwartungen gestellt. Sie sollen unter anderem die Beschäftigten motivieren, Controlling durchführen, sich um Innovationen kümmern, die Beschäftigten ansprechend informieren und qualifizieren, inner- und überbetrieblich kommunizieren. Die UMBs sehen sich meist selbst als Motor für den betrieblichen Umweltschutz und werden auch als solche von den Beschäftigten wahrgenommen.

\section{Interne Information und Kommunikation}

Interne Information ist Voraussetzung für jede Art von Beteiligung. Die zentrale Frage lautet: Welche Form der Information, möglich wären unter anderem Umwelterklärungen, die Mitarbeiterzeitung, das Schwarze Brett oder das Einzelgespräch, ist für welche Zielgruppe sinnvoll? Es existiert eine Vielfalt von Maßnahmen zur Förderung der innerbetrieblichen Kommunikation (2). Trotzdem lautet der zentrale Befund: die Potenziale der innerbetrieblichen Kommunikation werden nicht ausgeschöpft. Dabei spielt es keine Rolle, ob es um Fragen des Umweltschutzes, des Vorschlagswesens, Unternehmensziele oder elementare Arbeitsprozesse geht.

Die Durchdringung des UMS in die übrigen Geschäftsprozesse und die gesamte Organisationsstruktur weist Schwachstellen auf. Typischer Befund ist, dass Umweltschutz im technischen, produzierenden Bereich angesiedelt ist und manche
Unternehmensbereiche nichts mit dem UMS zu tun haben, zum Teil auch gar nichts davon wissen. Davon sind vor allem Verwaltung, Marketing, Vertrieb, aber auch Einkauf und Produktentwicklung betroffen.

Eine systematische Information der Beschäftigten über umweltbezogene Themen findet in wenigen Betrieben statt und weist selbst dort häufig Lücken auf. Damit ist die zentrale Grundlage und Voraussetzung für Beschäftigtenbeteiligung nur unzureichend gegeben. In der Regel werden Umweltinformationen auf der Leitungsebene, beim UM-Beauftragten und in den entsprechenden Gremien und Teams gebündelt.

Betriebsinterne Umweltdaten sind oft zumindest theoretisch zugänglich. Sie werden in unterschiedlicher Frequenz durch den UM-Beauftragten vorgelegt und in den entsprechenden Gremien erläutert. Allerdings werden sie selten strategisch als Kennzahlen genutzt oder anwendungsorientiert und verständlich für die Beschäftigten aufbereitet. Arbeitsplatzbezogene Umweltinformationen liegen in den wenigsten Betrieben vor. Produktbezogene Umweltinformation findet sich meist nur dort, wo die ökologische Qualität der Produkte im Vordergrund steht.

Für die Informationsvermittlung in KMU werden eher konventionelle Wege genutzt. Die Unterweisung spielt eine zentrale Rolle. Daneben gibt es schriftliche Formen der Information wie das Schwarze Brett, aber eine geringe Verbreitung von interaktiven Kommunikationsformen.

\section{Beschäftigten- und Betriebsrats- beteiligung}

Obwohl die aktive Beteiligung der Beschäftigten an den Umweltmanagementprozessen eine wesentliche Komponente erfolgreicher Umsetzung ist, werden die Möglichkeiten einer effektiven Beschäftigtenbeteiligung nicht ausgeschöpft. Die Motivation der MitarbeiterInnen in KMU für Umweltschutzbelange wird in vielen Untersuchungen als Erfolgsbedingung herausgestellt. Sie wird im Allgemeinen aber nicht als zentraler Problembereich gesehen, da in den evaluierten Beispielen Motivation und Umweltbewusstsein der Beschäftigten gleich bleibend positiv bewertet werden. Häufig wird auf die Notwendigkeit individuellen Engagements der MitarbeiterInnen verwiesen, das nötig sei, um ökologische Verbesserungen effizient und dauerhaft zu implementieren. Dies erfordert eine Qualifikationsanstrengung, die oftmals aus Kosten- und Personalmangel nicht in Angriff genommen wird. In KMU werden deutliche Män- 


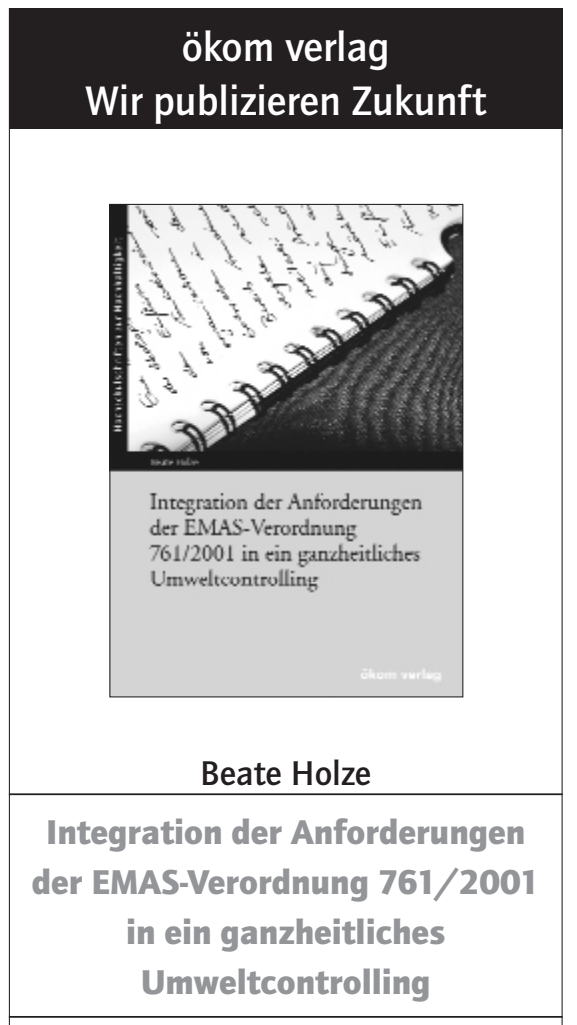

Seit „Basel II" die Eigenkapitalregeln neu definiert, werden Kreditkosten an der Bonität der Kunden ausgerichtet. Dabei achten einige Banken neben ökonomischen auch auf ökologische und soziale Kriterien. Unternehmen, die ihre Entwicklung am Leitbild der Nachhaltigkeit ausrichten, sind hier im Vorteil. Beate Holze formuliert einen Ansatz für ganzheitliches Umweltcontrolling, der sich an der EU-Verordnung 761/2001 „Eco Management and Audit Scheme" (EMAS II) orientiert. Sie erläutert Ausgangsbedingungen, Ablauf und Aufbau der Verordnung und zeigt, wie das Umweltcontrolling modifiziert werden muss, um den Anforderungen von EMAS II zu genügen. Am Beispiel einer Recyclinganlage wird ihr Ansatz ge-testet und bewertet.

Beate Holze

ökom verlag, München 2003

253 Seiten, 19,80 € (zzgl. Versandkosten) ISBN 3- 936581-18-5

\section{Erhältlich bei}

CONSODATA ONE-TO-ONE

Semmelweisstraße 8, D-82152 Planegg

Fon $++49 /(0) 89 / 857$ 09-155

Fax $++49 /(0) 89 / 857$ 09-131

kontakt@oekom.de gel bei der Qualifizierung der Beschäftigten festgestellt: es besteht die Gefahr, dass nach erfolgter Zertifizierung ein Stillstand in den Anstrengungen für eine kontinuierliche ökologische Verbesserung der Produktionsabläufe eintritt, weil die damit bislang betrauten Einzelpersonen sich anderen Aufgaben zuwenden oder aus anderen Gründen nicht weiter zur Verfügung stehen. Es gibt nur vereinzelt dauerhafte Strukturen, in denen die relevanten Personen hierarchieübergreifend an Problemlösungen arbeiten.

Nach einer Studie des Bonner Instituts für Mittelstandsforschung verfügen lediglich vier Prozent der Betriebe mit weniger als 20 Mitarbeitern über einen Betriebsrat, lediglich 16 Prozent der Unternehmen mit 21 bis 50 Beschäftigten, aber bereits 67 Prozent der Unternehmen mit 51 bis 500 Beschäftigten haben einen Betriebsrat. Kleinbetriebe kann man folglich zutreffend als ,betriebsratsfreie Zonen" definieren

Hier kann das novellierte Betriebsverfassungsgesetz (BetrVG) Abhilfe schaffen, indem es gerade die Möglichkeit der Bildung von Interessenvertretungen in Kleinbetrieben erleichtert. Auch der Umweltschutz ist als Gegenstandsbereich der Mitbestimmung für Betriebsräte in der Novellierung festgeschrieben worden. Dabei handelt es sich eher um ein Informationsrecht, das lediglich Mitwirkung aber nicht echte Mitbestimmung meint. Bei den Umweltmanagementpionieren hat die Novellierung wenig Auswirkungen. Eine aktive Teilnahme von Betriebsräten durch Mitarbeit in Gremien findet in etwa 50 Prozent der Betriebe statt, in einem Drittel der Betriebe sind Arbeitnehmervertreter informiert und unterrichtet, in einem Fünftel werden die Betriebsräte überhaupt nicht beteiligt. Bei der aktiven Teilnahme wurden durchweg positive Erfahrungen in der Zusammenarbeit gemacht, wobei als zentrales Handlungsmotiv die Arbeitsplatzsicherung gelten kann.

\section{Partizipation stärkt Ökologie}

Analysiert man die betrieblichen Umsetzungsprozesse, so lässt sich vor allem positiv herausarbeiten, dass in letzter Zeit die Bedeutung der internen Faktoren erkannt wurden. Partizipative Methoden und Instrumente zur Selbstevaluation oder Entwicklungsfähigkeit von Unternehmen im Bereich UMS werden entwickelt und angewandt (3). Der Umweltschutz wird nicht mehr nur als „Chefsache“ verstanden. Trotzdem bleiben die Potenziale der Beteiligung von Betriebsräten und Beschäftigten oft ungenutzt. Fehlende zielgruppenspezifische Informationen, Blockadetendenzen durch möglichen innerbetrieblichen Machtverlust und Angst vor neuen Themen und Strukturen sowie ein geringer Stellenwert von Qualifizierung in KMU, tragen zu dieser Situation bei.

Partizipation braucht Zeit für Weiterbildung, Lernen, Dialoge und Gestaltungsversuche. Die übliche Beschränkung von Beteiligungsprozessen auf die betriebliche Arbeitsgestaltung, Organisationsentwicklung oder auf die Technikgestaltung ist überholt. Beteiligung muss in Wertschöpfungsketten, in Unternehmensnetzwerken und an den Orten unternehmerischer Innovation organisiert werden. Denn wenn es Beteiligungsprozessen nicht gelingt, in den Bereich industrieller Innovation vorzustoßen, zum Beispiel der Produktentwicklung, dann wird es sich immer um eine nachhinkende Beteiligung nach Vollendung der Fakten handeln.

\section{Anmerkungen}

(1) Das ergab eine aktuelle Befragung von 30 Öko- und Umweltmanagementpionieren, die vom Klaus Novy Institut im Rahmen eines von der Hans Böckler Stiftung geförderten Projektes mit dem Titel "UMS und IPP als Gestaltungsfelder für KMU und Träger der Mitbestimmung" durchgeführt wurde. (2) Vgl. Klemisch, H.: Umweltmanagement und ökologische Produktpolitik - Partizipation betrieblicher und gesellschaftlicher Akteure an Ökologisierungsprozessen in Unternehmen und Branchen. Dissertation. München (im Erscheinen).

(3) Aus der Umweltmanagementpraxis heraus werden Instrumente nachhaltigen Wirtschaftens wie SAFE entwickelt oder neve Managementinstrumente wie die Balanced Scorecard erweitert. Vgl. Baedecker C./ Heuer P./ Klemisch H./ Rohn H.: Handbuch zur Anwendung von SAFE. Wuppertal 2003 sowie Schaltegger S./ Dyllick, T (Hg.): Nachhaltig managen mit der Balanced Scorecard. Wiesbaden 2002.

\section{Der Autor}

Dr. Herbert Klemisch ist Leiter des Referats Arbeit und Umwelt im Klaus Novy Institut. Kontakt: Klaus Novy Institut, Annostr. 27, 50678 Köln. Tel. 0221-93120715, Fax 022193120720, E-Mail: herbert.klemisch@kni.de 
(c) 20I0 Authors; licensee IÖW and oekom verlag. This is an article distributed under the terms of the Creative Commons Attribution Non-Commercial No Derivates License (http://creativecommons.org/licenses/by-nc-nd/3.o/), which permits unrestricted use, distribution, and reproduction in any medium, provided the original work is properly cited. 